\title{
Note on $\gamma$-Matrices Efficient at an Isolated Point
}

\author{
By P. Vernes
}

(Received 2nd September 1948. Read 5th November 1948.)

In a recent paper ${ }^{1} \gamma$-matrices were constructed summing the binomial series at isolated points to its right value. It is assumed that the reader is able to refer to this paper and is familiar with its notation and terminology.

The object of this note is to give the construction of $\gamma$-matrices which sum the binomial series at an isolated point to an arbilrary given value, while inside the circle of convergence the generalised sum is necessarily the right value.

We first consider the series $\Sigma z^{k}$ at $z=z_{0}$, where $\left|z_{0}\right|>1$. If $a$ is an arbitrary complex number, and $b=a-1 /\left(1-z_{0}\right)$, we construct the matrix

$$
G \equiv\left(g_{n, k}\right), \quad n, k=0,1,2, \ldots,
$$

given by $g_{n, k}=1$ for $k<n,=1 /\left(1-z_{0}\right)$ for $k=n,=b / 2^{k-n} z_{0}{ }^{k}$ for $k>n$.

Obviously $G$ is a $\gamma$-matrix, since $g_{n, k} \rightarrow 1$ as $n \rightarrow \infty$, and

$\sum_{k}\left|g_{n, k}-g_{n, k+1}\right|<1+2 /\left|1-z_{0}\right|+2|b| \Sigma\left|2 z_{0}\right|^{-k} \quad$ for every $n$.

Applying $G$ to the series $\Sigma z_{0}^{k}$, we have

$$
\begin{aligned}
& \begin{aligned}
S_{n}=\sum_{k} g_{n, k} z_{0}^{k} & =1+z_{0}+z_{0}^{2}+\ldots+z_{0}^{n-1}+z_{0}^{n} /\left(1-z_{0}\right)+b / 2+b / 4+\ldots \\
& =1 /\left(1-z_{0}\right)+b=a,
\end{aligned} \\
& \text { so that } S_{n} \rightarrow a
\end{aligned}
$$

For other values of $z, S_{n}(z)=\sum_{k} g_{n, k} z^{k}$ converges only if $|z|<2\left|z_{0}\right|$, and then

$$
S_{n}(z)=\frac{1}{1-z}+\frac{\left(z_{0}-z\right) z^{n}}{(1-z)\left(1-z_{0}\right)}+\frac{b z^{n}+1 z_{0}}{\left(2 z_{0}-z\right) z_{0}^{n+1}}
$$

which tends to a limit only if $z=z_{0}$ or $|z|<1$.

$1 \mathrm{P}$. Vermes, "On $\gamma$-matrices and their application to the binomial series," Proc. Ealinburgh Math. Soc. (2), 8 (1947), 1-13 (11-13). 
In the above construction we used the matrix $H\left(1, z_{0}\right)$ of the previous paper, replacing its zero elements by suitable numbers. Given the series $\Sigma c_{k} z^{k}$ representing $(1-z)^{-p}$, where $p$ is a positive integer, we replace the zero elements of the matrix $H\left(p, z_{0}\right)$ in a similar way. We then have

$g_{n, k}=\left(1-z_{0}\right)^{-p} \sum_{j \rightarrow 0}^{n-k}\left(\begin{array}{l}p \\ j\end{array}\right)\left(-z_{0}\right)^{j}$ for $k \leqq n, \quad=b / c_{k} z_{0}{ }^{k} 2^{k-n}$ for $k_{.}^{\prime}>n$, where $b \doteq a-1 /\left(1-z_{0}\right)^{p}$, and $G$ has the required properties.

Birkbeck College,

UNIVIRSITY OF LONDON. 